\title{
The Effect of Inhaled Gases on Ultrasound Contrast Agent Longevity In Vivo
}

\author{
Malak Itani, ${ }^{1,2}$ Robert F. Mattrey ${ }^{1,3}$ \\ ${ }^{1}$ Department of Radiology, UC San Diego, 200 W. Arbor Drive \#8749, San Diego, CA 92103-8749, USA \\ ${ }^{2}$ Department of Radiology, American University of Beirut, Beirut, Lebanon \\ ${ }^{3}$ Moores UCSD Cancer Center, La Jolla, CA, USA
}

\begin{abstract}
Purpose: The purpose of this study is to investigate the effect of the inhaled gas used alongside isoflurane in the anesthetization of small animals on the time-intensity curves (TICs) acquired from ultrasound contrast agents-microbubbles.

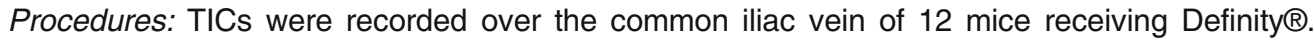
Animals were anesthetized with isoflurane, the ventilator was driven by medical air (MA), then in random order, the driving gas was changed for $3 \mathrm{~min}$ to: $\mathrm{MA}$ (control); pure oxygen $\left(\mathrm{O}_{2}\right) ; \mathrm{O}_{2}+$ perfluorohexane $\left(\mathrm{PFH}: \mathrm{O}_{2}\right)$; or $\mathrm{O}_{2}$ +octafluoropropane $\left(\mathrm{OFP}: \mathrm{O}_{2}\right)$, the perfluorocarbon $(\mathrm{PFC})$ in Definity, followed by a return to MA 3 min later.

Results: The mean slope of signal decay was $-0.47,-1.05,-1.16$, and -1.42 video-intensity units/s for MA, OFP: $\mathrm{O}_{2}, \mathrm{PFH}: \mathrm{O}_{2}$, and $\mathrm{O}_{2}$, respectively; MA had the slowest decay $(p<0.0001)$. Both PFC mixtures had slower signal decay than $\mathrm{O}_{2}$, but only OFP: $\mathrm{O}_{2}$ was significant $(p<0.01)$. When MA was used immediately following dosing, slope gradually decreased $(p=0.032)$ and was two times slower by the fourth injection $(p=0.012)$.

Conclusions: Microbubble kinetics are closely associated with the driving gas for inhaled anesthesia. MA has the least effect and should be used when inhaled anesthesia is used. Furthermore, when animals are given multiple injections in the same session, microbubbles last longer with subsequent injections.
\end{abstract}

Key words: Contrast-enhanced ultrasound, Microbubbles, Definity, Gas anesthesia, Mice, Oxygen, Medical air, Microbubble quantification, Microbubble half-life

\section{Introduction}

Oontrast-enhanced ultrasound (CEUS) is gaining increas$\checkmark$ ing importance in clinical and research applications. The microbubble-based ultrasound contrast agents (UCAs) used to enhance tissues differ dramatically from magnetic resonance and x-ray computed tomography agents in that ultrasound is extremely sensitive to microbubbles (MBs) and is capable of detecting a single moving MB [1], allowing for sub-trace doses, and, owing to their $1-3-\mu \mathrm{m}$ size, MBs remain within the vascular space. In addition to their

Correspondence to: Robert F. Mattrey; e-mail: rmattrey@ucsd.edu promising diagnostic use, MBs can be used to increase local drug and gene delivery for more effective therapy. These facts have resulted in rapid expansion of CEUS clinical applications, and have accelerated pre-clinical research to develop potential uses in molecular imaging-based diagnostic and therapeutic techniques.

Cancer detection with targeted MBs is a promising preclinical application that is being translated to the clinic. Much of the pre-clinical effort has focused on rodent models of tumor angiogenesis, ischemia, thrombosis, and inflammation [2]. For studies that assess perfusion [3], receptor density [4], and differential contrast characteristics [5], MB signal quantification and kinetics in the region of interest that is typically monitored by acquiring a time-intensity curve (TIC) are critical . 
While the TIC is crucial for studying kinetics in a region of interest, the observed kinetics include, in addition to local physiologic indices, MB loss from the systemic circulation caused by MB elimination and MB destruction by ultrasound itself. MB formulation affects both kinetics and the MBs' sensitivity to ultrasound, causing different elimination rates [68]; however, MB elimination is also affected by the inhaled gases, particularly oxygen $\left(\mathrm{O}_{2}\right)$, used when performing in vivo studies [9-11]. Former studies focused on Albunex and Optison, which have albumin shells, and Albunex is air filled. Fortunately, the majority of human studies and the animal studies done with injectable anesthesia are performed with the subject breathing room air. In few patients' studies and in the majority of rodent studies, imaging is performed with $100 \% \mathrm{O}_{2}$ inhalation that negatively impacts MB survival [11]. Gas anesthesia is preferred, particularly in small animals, because injectable anesthesia impacts homeostasis and provides a narrow safety margin [12].

Gas anesthesia requires a pressurized gas to drive the ventilator. Further, CEUS studies are being performed in rodents with gas anesthesia that use signal enhancement and/ or TICs as end-points without mentioning or controlling the driving gas used [4, 13-18]. It is therefore prudent to define the influence of inhaled gases on MB survival within the vascular space in rodents.

The primary purpose of this study was therefore to investigate in mice the effect of the pressurized gas used during gas anesthesia on MB survival. There were two secondary aims for this study: (1) Since MBs' perfluorocarbon (PFC) gas core is exhaled because of the lower partial pressure gradient between the blood and alveolar space [19, 20], we hypothesized that adding PFC vapor to the inhaled gas could slow the PFC loss and increase MB survival. (2) Since many experiments include multiple injections of either the same MB formulation or targeted $v s$. non-targeted MBs, and since PFC can remain in the circulation and lungs for $1-2 \mathrm{~h}$ [20], we also aimed to assess whether a MB injection whose ultrasound effect had returned to baseline would affect MB survival on subsequent injections.

\section{Materials and Methods}

\section{Inhaled Gases}

We used four different pressurized gases to drive the ventilator to administer isoflurane anesthesia. Once the experiment began, the isoflurane and gas flow were maintained constant. We used medical air (MA); pure $\mathrm{O}_{2}$; a mixture of $\mathrm{O}_{2}$ and octafluoropropane (OFP), the gas core used in Definity ${ }^{\circledR}$, the only commercially available MB in the USA; and a mixture of $\mathrm{O}_{2}$ and perfluorohexane (PFH). $\mathrm{PFH}$ was used to determine whether any PFC could affect the survival of an OFP-filled MB.
MA is composed of $5 \% \mathrm{CO}_{2}, 21 \% \mathrm{O}_{2}$, and $74 \%$ nitrogen (Airgas West, Lakewood, CA, USA). The $\mathrm{O}_{2}$ was $\geq 99 \%$ pure (Praxair, Inc., Danbury, CT, USA). The PFCs were $\geq 98 \%$ pure (SynQuest Labs, Inc., Alachua, FL, USA). They were mixed with $\mathrm{O}_{2}$ in a sealed plastic bag and then pressurized to flow through the anesthesia system. Being a gas, OFP was mixed directly with pure $\mathrm{O}_{2}$. Since $\mathrm{PFH}$ is liquid at room temperature $\left(\mathrm{BP}=55^{\circ} \mathrm{C}\right)$, pure $\mathrm{O}_{2}$ was percolated through $\mathrm{PFH}$ using two diffusers connected in series to saturate $\mathrm{O}_{2}$ with $\mathrm{PFH}$ vapor. The generated PFC: $\mathrm{O}_{2}$ mixtures were analyzed by gas chromatography (HP-GC-5890 Series II ${ }^{\circ}$, Hewlett-Packard Company, Houston, TX, USA) with a GS-Q column. The ratio of OFP in the OFP: $\mathrm{O}_{2}$ mixture ranged from $50 \%$ to $70 \%$ and $\mathrm{PFH}$ in the $\mathrm{PFH}: \mathrm{O}_{2}$ mixture from $51 \%$ to $66 \%$ by volume.

\section{Animal Model}

The experiment used 15 7- to 9-week-old NIH Swiss mice (Harlan Laboratories). All animals were induced using MA and isoflurane (1.5-2\%) and placed supine on a heated table integrated into the Visualsonics Vevo770 small-animal imaging system (VisualSonics, Toronto, Ontario, Canada). The groin and neck were clipped and the remaining hair removed with a depilatory cream. The right jugular vein was surgically exposed for direct MB injection. All procedures were pre-approved by the Institutional Animal Care and Use Committee.

\section{UCA and Injection}

Definity (lot\# 4579, distributed by Lantheus Medical Imaging) was used for all experiments. Each vial was prepared as instructed by the manufacturer by shaking for $45 \mathrm{~s}$ using the apparatus provided by the manufacturer (Bristol-Myers Squibb Medical Imaging, Inc., North Billerica, MA, USA). Microbubbles within the vial were resuspended by hand agitation for $10 \mathrm{~s}$ just before dosing. Since each mouse was injected four times per session, all doses were withdrawn from the same vial within $4 \mathrm{~h}$ and the vial discarded. Injections of $1 / 20$ dilution were prepared by mixing $0.01 \mathrm{~mL}$ of Definity with $0.19 \mathrm{~mL}$ of normal saline in a $0.3-\mathrm{mL}$ syringe using a 28 -gauge needle. The suspension in the syringe was prepared immediately prior to each injection.

\section{Image Acquisition}

The imaging protocol is shown in Fig. 1. The 40-MHz transducer (RMV706) was used to image the common iliac veins in the transverse plane using standard B-mode. A region-of-interest was positioned within the vein and was not moved throughout the entire observation period. Video signals were measured by the instrument in real 


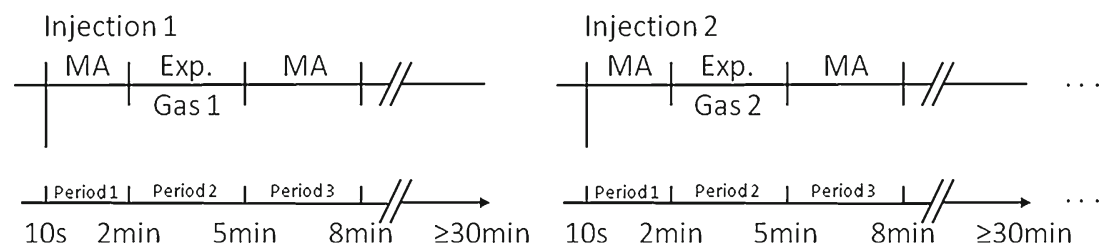

Fig. 1. Imaging protocol of each mouse. Injections were given when mice were breathing medical air (MA). At 2 min after injection, they were switched to the Exp. Gas that was either $\mathrm{MA}, \mathrm{O}_{2}, \mathrm{OFP}: \mathrm{O}_{2}$, or $\mathrm{PFH}: \mathrm{O}_{2}$ - given in random order, and the signal monitored for $3 \mathrm{~min}$. They were then returned to MA and monitored for an additional 3 min. Each mouse received four injections of $0.1 \mathrm{~mL}$ of $1 / 20$ dilution of Definity at least $30 \mathrm{~min}$ apart when signal returned to baseline.

time on each frame, acquired at five frames per second. When at least $10 \mathrm{~s}$ were recorded at baseline while the animal was still on MA, $0.1 \mathrm{~mL}$ of the diluted contrast was administered slowly over $10-15 \mathrm{~s}$ and the signal recorded for $2 \mathrm{~min}$. At 2 min post-dosing, the pressurized gas was changed to either: (a) MA, which served as control; (b) $\mathrm{O}_{2}$; (c) $\mathrm{O}_{2}+\mathrm{OFP}\left(\mathrm{OFP}: \mathrm{O}_{2}\right)$; or (d) $\mathrm{O}_{2}+\mathrm{PFH}$ $\left(\mathrm{PFH}: \mathrm{O}_{2}\right)$, selected at random using a random number generator, and the signal recorded for an additional $3 \mathrm{~min}$. The pressurized gas was then returned to MA for an additional $3 \mathrm{~min}$ of signal recording. The video signal within the vein for the entire 8-min observation period was saved as a numerical data file for analysis. Signal was monitored until it returned to baseline or for $30 \mathrm{~min}$ after contrast administration, whichever was greater. Each mouse received four injections within the same experimental session, with one injection for each pressurized gas.

\section{Data Reduction}

Three animals died before completing all four injections, resulting in 12 complete mice (48 injections) whose data was used for analysis. Video signals in the iliac vein were plotted over time for each of the three observation periods for each experimental gas (Fig. 2). Because the frame-to-frame signal was highly variable due mostly to pulsatile motion from the adjacent artery, a 50-point moving average (10 s of data) was calculated and plotted. A linear regression was fitted to

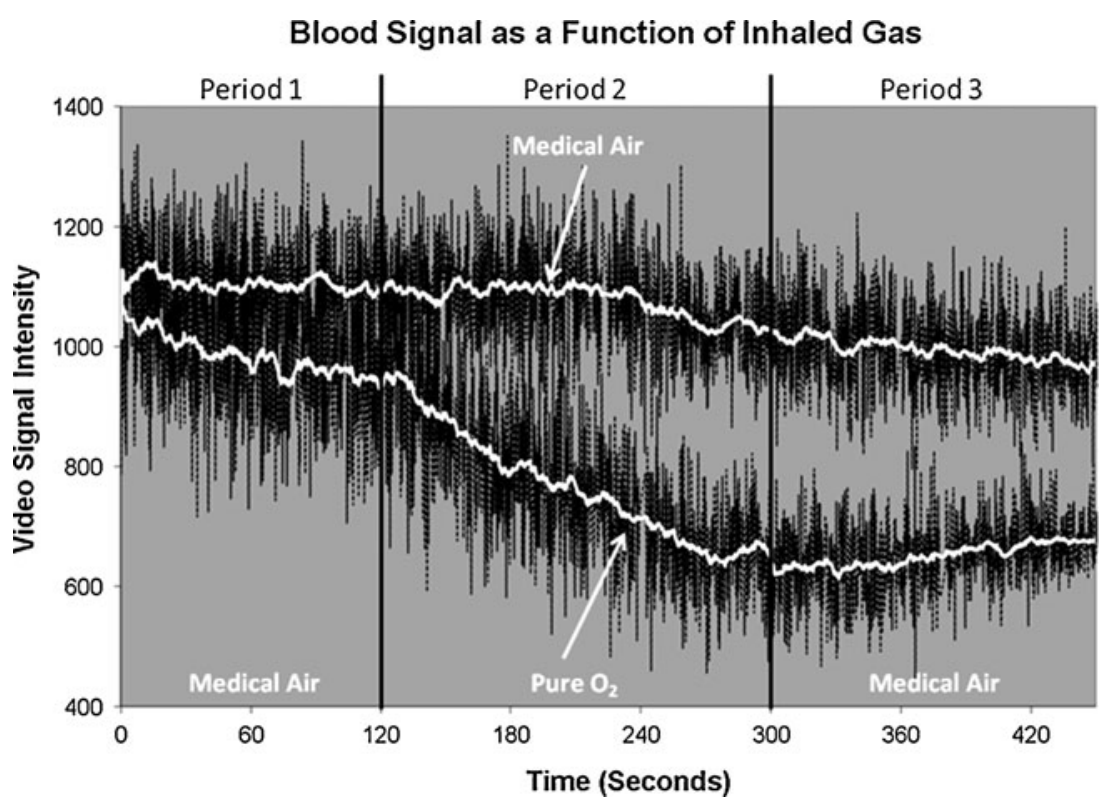

Fig. 2. Typical example of venous signal from the same mouse following two separate injections when it was breathing MA during periods 1 and 3 of both injections and MA (above curve) or pure $\mathrm{O}_{2}$ (bottom curve) during period 2. The high frame-to-frame signal variation was smoothed by calculating a 50-point (10 s of data) moving average (thick white line). The data from the moving average was used to fit a regression line to define the slope of signal decay for each of the three observation periods. Note that the decay curve in period 2 when the animal was breathing pure $\mathrm{O}_{2}(-1.82$ units/s) is four times faster than when breathing MA (-0.43 units/s) in this animal. Also note that when this animal was returned to MA after $\mathrm{O}_{2}$, signal increased. 
the moving average, excluding the first $10 \mathrm{~s}$ of each period to allow the new gas to fill the alveoli. The slope of signal decay was calculated for each of the three observation periods following each injection. Therefore, each mouse contributed 12 slopes in total: four for MA in period 1 (MAbaseline); one each for $\mathrm{MA}, \mathrm{O}_{2}, \mathrm{OFP}: \mathrm{O}_{2}$, and $\mathrm{PFH}: \mathrm{O}_{2}$ for period 2; and, finally, four additional slopes for MA in period 3 (MA-post). Although signal decay is exponential, the high frame-to-frame variation due to the small region-ofinterest in the iliac veins and motion did not allow accurate fitting.

\section{Data Analysis}

The slopes from all mice for each of the three periods and for each gas used were averaged and the standard error of the mean (SEM) calculated. All data analysis was performed using NCSS2007. A two-way analysis of variance (ANOVA) was used to evaluate whether the slope of the signal decay was significantly different between the first, second, and third observation periods after each injection and for each of the experimental gases between injections. The gas and the order it was given (first, second, third, or fourth) to each mouse were used as the independent variables. A $p$ value $\leq 0.05$ was considered significant. When a significant effect was detected by ANOVA, a pair-wise two-tail Student's $t$ test was then carried out. We used paired statistics when comparing the slopes among the observation periods and unpaired statistics when comparing the slopes between the gases used in period 2. Fisher's least significant difference was used as a correction method for the multiple comparison tests.

\section{Results}

When comparing the effect of each gas on the rate of signal decay in the second observation period, the order in which the gas was given to each mouse had no effect $(p=0.13)$; however, there was a marked difference in slopes among the gases used $(p<0.0001)$. The slopes and their pair-wise comparisons are shown in Fig. 3. Note that the least decay was observed when animals were breathing MA $(p<0.001)$, which was three times slower than when they were breathing pure $\mathrm{O}_{2}(p<0.0001)$. The addition of slightly more than $50 \%$ by volume $\mathrm{PFC}$ to $\mathrm{O}_{2}$ slowed MB decay. While the addition of PFH to $\mathrm{O}_{2}$ slowed the decay rate by $19 \%$, the difference was not statistically significant $(p=0.126)$. However, when the PFC was the same as that filling the MB core, signal decay slowed by $26 \%(p=0.006)$.

Signal decay when MA was used throughout the three observation periods in the same mouse was identical $(-0.41 \pm$ 0.08 for MA pre, $-0.47 \pm 0.07$ for MA, and $-0.49 \pm 0.04$ for MA post, $p=0.41$ ). Comparing the MA-post signal decay to the MA-pre decay after mice were breathing either O2, OFP: $\mathrm{O} 2$ or $\mathrm{PFH}: \mathrm{O} 2$, the decay rate was 2.6 times slower (MAbaseline; $-0.55 \pm 0.05$ vs. $-0.22 \pm 0.06 ; p=0.0003$ ), even though animals were breathing MA in both periods; this suggests a partial signal recovery when the animal was returned to breathing MA. This is illustrated in Fig. 4, where theoretical TICs were constructed based on the mean slope

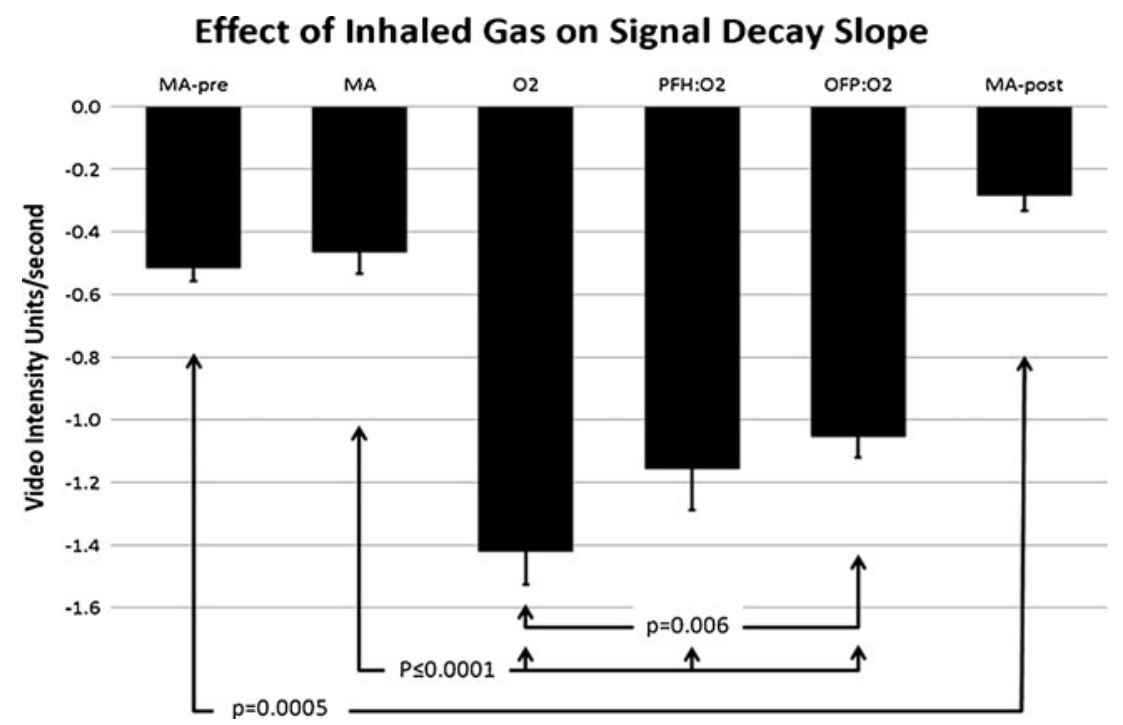

Fig. 3. The average slope and SEM are shown for MA at baseline (MA-pre) (period 1) and MA-post (period 3), and for all four individual gases used in period 2. Since MA pre- and post- were acquired with every injection, the mean and SEM were calculated from all 48 injections. The values for the individual gases are based on the 12 injections, one from each mouse. Pairwise comparisons are as shown. All other paired comparisons that are not shown were not statistically significant. 


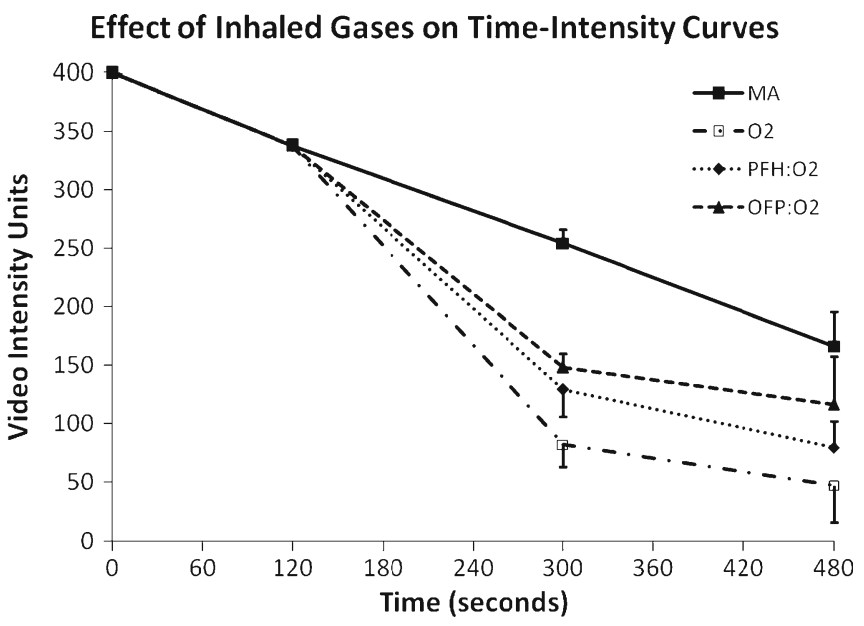

Fig. 4. Theoretical time-intensity curves constructed by starting from an arbitrary 400 video-intensity units and applying the mean slope associated with MA-baseline, each gas used in period 2, and the slope during MA-post following each of the four gases. The error bar reflects the error calculated based on the SEM for each of the three periods for each gas. Since the slopes during MA-baseline for each gas were not statistically different, the mean MA-baseline slope and SEM for all injections were used for clarity. Note that when MA was used in period 2, the decay curve throughout all three observation periods is nearly linear. The decay rate in period three while the animals are breathing MA following the use of gases other than MA in period 2, slows by 2.6 times $(p=0.0003)$.

and SEM for each of the three periods for each of the four gases used.

Although all animals were breathing MA during the first observation period, analysis of signal decay during this period revealed that while the gas to be used in period 2 had

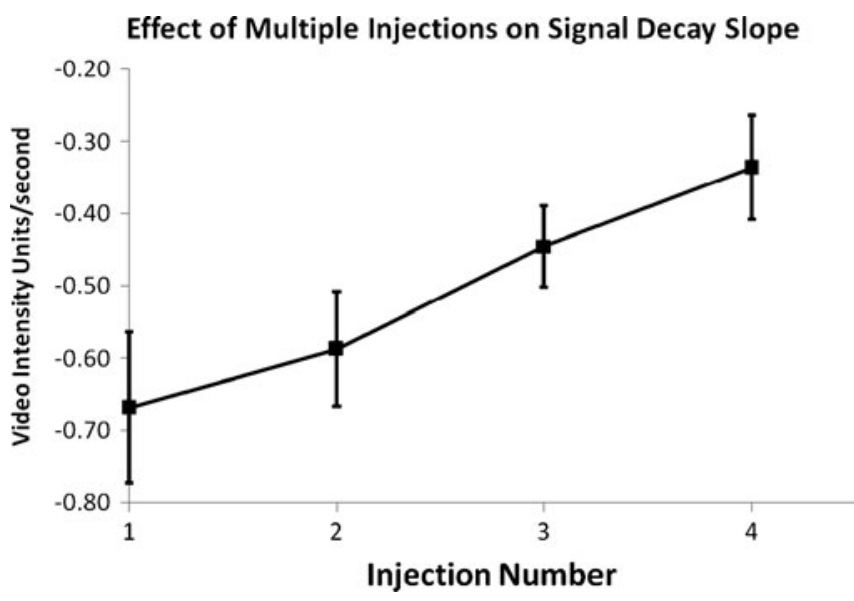

Fig. 5. Slope of signal decay \pm SEM while the animal is breathing MA immediately following contrast administration in period 1 prior to switching the gas. The slope is plotted against the injection order of four consecutive injections. Note that the signal is lost at a slower rate, with subsequent injections doubling microbubble survival by the fourth injection. no effect on slope as would be expected $(p=0.146)$, the injection order had a significant effect $(p=0.032)$. The slope during the first observation period and the SEM as a function of injection order are shown in Fig. 5. Note that the fastest signal decay occurred after the first injection. Signal decay slowed with subsequent injections and was two times slower by the fourth injection $(p=0.012)$.

\section{Discussion}

The results of this study show that inhaled gases have a profound effect on MB survival in the systemic circulation and is in concordance with the results recently reported by Mullen et al. who modeled the effect of ambient gases on the rate of change of MB size [21]. The Definity used in this study - the only ultrasound contrast agent clinically available in the USA - is a suspension of MBs filled with pure OFP (C3F8), a PFC with low plasma solubility. These MBs initially swell when administered intravenously, as the OFP acts as an osmotic agent driving in oxygen $\left(\mathrm{O}_{2}\right)$ and nitrogen $\left(\mathrm{N}_{2}\right)$ into the MB $[8,22]$. As MBs that have equilibrated with venous blood traverse the pulmonary circulation, gases within the MBs equilibrate with those filling the alveolar space. Not only will their $\mathrm{N}_{2}$ and $\mathrm{O}_{2}$ content exchange, but their PFC is lost to the alveoli and is subsequently exhaled [20]. Furthermore, the ambient blood and insonation pressures and the lipid composition of the $\mathrm{MB}$ shell will affect the multi-gas equilibriums as the MBs circulate through the circulation and as they pass through a region being insonated [7, 8, 21-23]. Ultimately, as the PFC vapor within the MB decreases, the surface tension combined with the ambient pressure, particularly when ultrasound peak pressure is added, the vapor pressure of the PFC is exceeded and the PFC becomes liquid and loses its ultrasound backscatter advantage [22, 24]. Relative to red blood cells, liquid PFC droplets are seven to eight times more reflective, while MBs are at least ten orders of magnitude more reflective $[25,26]$.

It was observed that when Albunex, the first agent approved for human use that used denatured albumin to encapsulate air, was injected intravenously into animals breathing nitrous oxide and $\mathrm{O}_{2}$, no signal was detected in the left ventricle [11]. With the animals still breathing $\mathrm{O}_{2}, \mathrm{MBs}$ reached the left ventricle when a $\mathrm{PFC}$ was added to the $\mathrm{MB}$ core [10]. In our study, MBs filled with pure PFC disappeared from blood three times faster when animals breathed $\mathrm{O}_{2}$ compared to medical air.

The $\mathrm{N}_{2}$ that makes up approximately $80 \%$ of the gas in the alveolar space and the gas dissolved in blood is not consumed by the organism, serving to stabilize the MB as was very recently shown by Mullen et al. [21], when they modeled gas transport across MBs. Our results also show that the loss of PFC to the alveolar space is a factor since the addition of PFC to pure oxygen in the inhaled gas increased MB survival. It appears that when OFP was added, MBs survived slightly longer in the circulation than when $\mathrm{PFH}$ was added ( $26 \%$ vs.. 19\%). This suggests that not only is 
PFC loss to the alveolar space a contributing factor, but the type of PFC used could also have an effect. While OFP that fills the MB core could be superior to PFH when it fills the alveolar space, it is possible that the PFC that is not consumed by the organism served the role of $\mathrm{N}_{2}$, and OFP was more effective than PFH in that function. However, the difference between OFP and PFH could also be due to the fact that PFH is liquid at room and body temperatures and could not achieve the same partial pressure as OFP. Although we used two stations to fully saturate the $\mathrm{O}_{2}$ gas with $\mathrm{PFH}$ vapor, it might not have been sufficient. Please note that the use of PFCs in this experiment was not intended as an approach to lengthen MB survival, but rather to gain insight into a possible mechanism of MB longevityt in vivo. It should also be noted that adding PFC to inhaled gases is costly, impractical, and much less effective than MA.

We noted in this study that the decay rates were identical when animals breathed MA throughout all three observation periods; however, when animals breathed $\mathrm{O}_{2}$ or either of the PFC mixtures in the second period, the decay rate during the first period was 2.6 times faster than in the third period. The example in Fig. 2, in fact, shows a positive slope in period 3 following $\mathrm{O}_{2}$ breathing, indicating not only slowing of $\mathrm{MB}$ decay but also signal recovery in this animal. Among all animals, however, there continued to be signal decay in the third period, albeit at a slower rate (see Fig. 4). This observation suggests that some MBs that were in the process of shrinking due to the absence of $\mathrm{N}_{2}$ re-inflated fully or partially when $\mathrm{N}_{2}$ was provided [22],

This study also showed that there was a detectable interference of one injection on MB survival on subsequent injections. Each mouse received four injections separated by at least $30 \mathrm{~min}$ or when blood signal had returned to baseline, whichever was longer. All injections were given when animals were breathing MA prior to switching the inhaled gas. Microbubble survival increased with subsequent injections and slightly more than doubled by the fourth injections. This is likely due in part to an increasing PFC level in the mouse from one injection to the next. When blood and exhaled gases were monitored in human subjects, perfluorobutane $\left(\mathrm{C}_{4} \mathrm{~F}_{10}\right)$ was still detectable at $2 \mathrm{~h}$ after injection [20]. Although OFP $\left(\mathrm{C}_{3} \mathrm{~F}_{8}\right)$ is more volatile than perfluorobutane and would likely be lost in a shorter period, it is expected that some OFP would still be present by 30 min to slow PFC loss. Further, PFC gas is heavier than air and will likely not wash-out rapidly from the lung, slowly decreasing the rate of PFC loss to the alveoli. Finally, as MBs collapse into liquid and become invisible on ultrasound $[8,22]$, their PFC is available to transfer to MBs because of the large partial pressure gradient between the liquid and gaseous phases, increasing MB survival.

\section{Conclusions}

Gas anesthesia with isoflurane is the preferred and the most commonly used method to anesthetize mice for imaging. With increasing number of studies using microbubble-based ultra- sound contrast in molecular imaging, it is critical to control for the effect of the pressurized gas used since it could have profound effects on MB survival and signal. This is particularly important in those studies that use tissue signal or timeintensity curves as end-points for efficacy. These studies should at least report the inhaled gas used. The fact that MA was the gas with the longest MB survival is advantageous, since MA is inexpensive, safe, and widely available. Since multiple injections are typically used in the same mouse to compare one formulation of MBs to another or one intervention to another, it is prudent to space the injections as far apart as is feasible to minimize the interference of one injection upon the other. Knowing this, it is also possible to design the experiment to control for this potential bias.

Acknowledgments. This study was supported in part by the NIH ICMIC P50-CA128346 and the NIH Roadmap R21-EB005360 grants. Special thanks to David Fisher (UCSD Dept. of Radiology) for his assistance in this study and for preparing and analyzing the gas mixtures; Eric Benson from Dr. Clifford Kubiak's laboratory (UCSD Dept. of Chemistry \& Biochemistry) for his assistance in performing gas chromatography; Dr. Karen Messer (Moores UCSD Cancer Center Biostatistics/Bioinformatics Shared Resource) for statistical analysis; and Emily Bass for editing the manuscript.

Conflict of interest disclosure. The authors declare that they have no conflict of interest.

Open Access. This article is distributed under the terms of the Creative Commons Attribution Noncommercial License which permits any noncommercial use, distribution, and reproduction in any medium, provided the original author(s) and source are credited.

\section{References}

1. Klibanov AL, Rasche PT, Hughes MS, Wojdyla JK, Galen KP, Wible JH Jr, Brandenburger GH (2004) Detection of individual microbubbles of ultrasound contrast agents: imaging of free-floating and targeted bubbles. Invest Radiol 39(3):187-95

2. Kaufmann B, Lindner J (2007) Molecular imaging with targeted contrast ultrasound. Curr Opin Biotechnol 18(1):11-6

3. Krix M, Krakowski-Roosen H, Kauczor H, Delorme S, Weber M (2009) Real-time contrast-enhanced ultrasound for the assessment of perfusion dynamics in skeletal muscle. Ultrasound Med Biol 35 (10):1587-95

4. Andonian S, Coulthard T, Smith AD, Singhal PS, Lee BR (2009) Realtime quantitation of renal ischemia using targeted microbubbles: in-vivo measurement of P-selectin expression. J Endourol 23(3):373-8

5. Chen L, Xu H, Xie X, Xie X, Xu Z, Liu G, Wang Z, Lin M, Lu M (2009) Intrahepatic cholangiocarcinoma and hepatocellular carcinoma: differential diagnosis with contrast-enhanced ultrasound. Eur Radiol 20 (3):743-753. doi:10.1007/s00330-009-1599-8

6. Ferrara K, Pollard R, Borden M (2007) Ultrasound microbubble contrast agents: fundamentals and application to gene and drug delivery. Annu Rev Biomed Eng 9:415-47

7. Gerber F, Waton G, Krafft MP, Vandamme TF (2007) Long lived microbubbles for oxygen delivery. Artif Cells Blood Substit Immobil Biotechnol 35(1):119-24

8. Kabalnov A, Bradley J, Flaim S, Klein D, Pelura T, Peters B, Otto S, Reynolds J, Schutt E, Weers J (1998) Dissolution of multicomponent microbubbles in the bloodstream: 2 Experiment. Ultrasound Med Biol 24(5):751-60

9. Porter T, Kricsfeld D, Cheatham S, Li S (1998) Effect of blood and microbubble oxygen and nitrogen content on perfluorocarbon-filled dextrose albumin microbubble size and efficacy: in vitro and in-vivo studies. J Am Soc Echocardiogr 11(5):421-5

10. Wible J Jr, Wojdyla J, Bugaj J, Brandenburger G (1998) Effects of inhaled gases on the ultrasound contrast produced by microspheres 
containing air or perfluoropropane in anesthetized dogs. Invest Radiol 33 (12):871-9

11. Wible JH Jr, Wojdyla JK, Bales GL, McMullen WN, Geiser EA, Buss DD (1996) Inhaled gases affect the ultrasound contrast produced by Albunex in anesthetized dogs. J Am Soc Echocardiogr 9(4):442-51

12. Donovan J, Brown P. (2001) Anesthesia. Curr Protoc Immunol Chapter 1 Unit 1.4.

13. Yankeelov T, Niermann K, Huamani J, Kim D, Quarles C, Fleischer A, Hallahan D, Price R, Gore J (2006) Correlation between estimates of tumor perfusion from microbubble contrast-enhanced sonography and dynamic contrast-enhanced magnetic resonance imaging. J Ultrasound Med 25(4):487-97

14. Lee D, Lyshchik A, Huamani J, Hallahan D, Fleischer A (2008) Relationship between retention of a vascular endothelial growth factor receptor 2 (VEGFR2)-targeted ultrasonographic contrast agent and the level of VEGFR2 expression in an in-vivo breast cancer model. J Ultrasound Med 27(6):855-66

15. Loveless M, Li X, Huamani J, Lyshchik A, Dawant B, Hallahan D, Gore J, Yankeelov T (2008) A method for assessing the microvasculature in a murine tumor model using contrast-enhanced ultrasonography. J Ultrasound Med 27(12):1699-709

16. Willmann J, Lutz A, Paulmurugan R, Patel M, Chu P, Rosenberg J, Gambhir S (2008) Dual-targeted contrast agent for US assessment of tumor angiogenesis in-vivo. Radiology 248(3):936-44

17. Stapleton S, Goodman H, Zhou Y, Cherin E, Henkelman R, Burns P, Foster $F$ (2009) Acoustic and kinetic behaviour of definity in mice exposed to high frequency ultrasound. Ultrasound Med Biol 35(2):296-307
18. Sullivan J, Wang B, Boesen E, D’Angelo G, Pollock J, Pollock D (2009) Novel use of ultrasound to examine regional blood flow in the mouse kidney. Am J Physiol Ren Physiol 297(1):F228-35

19. Morel D, Schwieger I, Hohn L, Terrettaz J, Llull J, Cornioley Y, Schneider M (2000) Human pharmacokinetics and safety evaluation of SonoVue, a new contrast agent for ultrasound imaging. Invest Radiol 35(1):80-5

20. Landmark KE, Johansen PW, Johnson JA, Johansen B, Uran S, Skotland T (2008) Pharmacokinetics of perfluorobutane following intravenous bolus injection and continuous infusion of sonazoid in healthy volunteers and in patients with reduced pulmonary diffusing capacity. Ultrasound Med Biol 34(3):494-501

21. Mullin L, Gessner R, Kwan J, Borden MA, Dayton PA (2009) An in-vivo evaluation of the effects of anesthesia carrier gases on ultrasound contrast agent circulation. ULTSYM 5441486:1290-1293

22. Kabalnov A, Klein D, Pelura T, Schutt E, Weers J (1998) Dissolution of multicomponent microbubbles in the bloodstream: 1 Theory. Ultrasound Med Biol 24(5):739-49

23. Kwan JJ, Borden MA (2010) Microbubble dissolution in a multigas environment. Langmuir 26(9):6542-8

24. Schutt EG, Klein DH, Mattrey RM, Riess JG (2004) Injectable microbubbles as contrast agents for diagnostic ultrasound imaging: the key role of perfluorochemicals. Angewandte Chemie International Edition 42:3218-3235

25. Mattrey RF, Steinbach GC (1991) Ultrasound contrast agents. State of the art. Invest Radiol 26(Suppl 1):S5-11, discussion S5

26. Ophir J, Parker KJ (1989) Contrast agents in diagnostic ultrasound. Ultrasound Med Biol 15(4):319-33 